\title{
Addressing patient treatment backlogs
}

\section{Dr Lynn Stephens}

Dento-legal advisor, BDA Indemnity

I is unlikely that dental practices are currently operating at their preCOVID-19 capacity. Some may still be restricting their services to minimal non-urgent treatment. It is now more than a year since dental practices were told to close because of the COVID-19 pandemic and many courses of treatment planned prior to March 2020 are still incomplete.

\section{Getting it finished}

With a significant proportion of the population having received their first vaccination, and infection rates decreasing again, patients may soon want to complete any postponed dental treatment.

Unfortunately, many patients do not appear to realise that dental practice is now very different. Expectations of what a practice is able to offer that might have been reasonable prior to lockdown might now be unreasonable. At BDA Indemnity we are currently advising dentists who have received complaints from patients who do not understand or wish to cooperate with new operating procedures. Some feel they should have been prioritised, whilst others feel they didn't receive the treatment they wanted in a timely manner.

In some cases, it has not been possible for patients to continue treatment with the same dentist they first saw. A number of clinicians have felt unable to return to work due to a personal vulnerability and may still be unaware when this will be possible. Some practices have sadly had to terminate the contracts of their Associates, and others may be working limited hours. Patients may also simply decide not to return to the practice they had previously attended.

\section{Dento-legal pitfalls}

$\rightarrow$ The returning patient may have a treatment plan that has yet to be started or may have been partially completed. If the original dentist is no longer available to see the patient there is a professional obligation for the practice to arrange continuity of care, even if the dentist who planned the treatment is no longer there.

$\rightarrow$ The patient's oral condition may or may not have changed in the intervening months. Whatever stage of treatment has been reached it is very important to undertake a comprehensive reassessment. Should things go wrong, it will be no defence to say that you didn't plan the treatment and were simply following the pre-existing treatment plan. It is your personal responsibility to ensure that the treatment plan is appropriate.

$\rightarrow$ The reassessment may result in a slightly revised or even a significantly different treatment plan than had originally been provided, depending upon the patient's condition and the new dentist's clinical opinion. In extreme cases, the original treatment plan may now be completely inappropriate.

$\rightarrow$ Try not to be critical of a plan with which you disagree. Remember that it is not at all unusual for two or more dentists examining the same patient to come up with significantly different treatment plans. Dentistry is not an exact science and a difference of clinical opinion does not mean that one clinician is correct and the other one is not.

$\rightarrow$ Greater problems may arise if the treatment has been partially completed or some payment made in advance. The patient may be pressurising for completion and may not take kindly to being told by a new dentist that the original treatment plan needs to be amended especially if this increases the cost.

$\rightarrow$ Even if there is no change to the patient's condition is the proposed treatment within the clinical capability of the new dentist? Indeed, it is not impossible that there is no-one in the practice who can now provide the treatment that is required. The practice should take steps to arrange a suitable referral. If the dentist who originally planned the treatment still works locally can an agreement be reached to complete the treatment with him/her? This is a situation the patient may be keen to pursue but can be fraught with difficulties and is a source of many disputes between clinicians and causes problems for patients where some payment has been made in advance

$\rightarrow$ If a dentist has to self-isolate for short periods due to a COVID-19 contact, it will usually be preferable to delay a complex treatment plan rather than have another dentist take over. If some urgent treatment is required in the meantime, care should be taken not to compromise the overall treatment plan by providing treatment that is irreversible such as an extraction. A phone discussion with the usual treating dentist may be beneficial, if this is possible.

\section{NHS treatment plans}

These are very likely to be restarted as a new course of treatment. Take care to comply with the claiming regulations if a transfer of care is required within a course of NHS treatment, either for the provision of urgent treatment or the completion of the course of treatment.

\section{Talk to the patient}

A detailed but empathetic explanation offered to the patient, and the options available, is the best way to achieve an amicable outcome. Some of this conversation may fall to practice staff who should document this within the patient records. From the dentist's point of view any changes to treatment plans must be explained carefully, especially when the changes are significant and could compromise the expected outcome, and again should be carefully documented. Patient consent can be defined as the voluntary and ongoing permission of the patient to receive a particular treatment. Claims often succeed when there is no evidence of the patient's consent to any changes in their treatment plan.

As in so many aspects of dentistry, the key to a satisfactory transfer of care is good communication. The time spent giving explanations will usually be time well spent. *

https://doi.org/10.1038/s41404-021-0709-4 